\title{
Cuidados de enfermería para recién nacido que padecen de ictericia patológica
}

\section{Nursing care for newborns suffering from pathologic jaundice}

\author{
Nairovys Gómez-Martínez \\ ua.nairovysgomez@uniandes.edu.ec \\ Universidad Regional Autónoma de los Andes, Ambato \\ Ecuador \\ https://orcid.org/0000-0003-1120-8408 \\ Evelyn Soraya Mesías-Núñez \\ ea.evelinsmn58@uniandes.edu.ec \\ Universidad Regional Autónoma de los Andes, Ambato \\ Ecuador \\ https://orcid.org/0000-0001-5493-6216
}

Recepción: 10 de agosto 2021

Revisado: 15 de septiembre 2021

Aprobación: 15 de noviembre 2021

Publicación: 01 de diciembre 2021 


\section{Sra. Editora:}

Se realizó un plan de cuidados de enfermería enfocado en la atención de recién nacido con ictericia patológica, abarcando las necesidades que debemos atender para así garantizar cuidados de enfermería enfocados en la mejora de la situación clínica del recién nacido. A través de la identificación de los beneficios se pudo aplicar el plan de cuidados de enfermería en paciente en estudio con Diagnóstico de ictericia patológica, enfocándonos en el cuidado directo mejorando así su atención y disminución de la estadía hospitalaria.

En la necesidad de Evitar peligros/seguridad, se observó una mejoría de 1 a 5 puntos, en los tres indicadores: temperatura de la piel, sensibilidad, pigmentación anormal, observando cambios significativos en el nivel de bilirrubina sérica $2 \mathrm{mg} / \mathrm{dL}$.

En la necesidad de Beber y comer, se observó una mejoría en cuanto 2 a 5 puntos, luego de la intervención de Enfermería al identificar el diagnóstico enfermero, patrón de alimentación ineficaz del lactante observando cambios notorios en el reflejo de succión del recién nacido siendo su peso al alta de 3414 gramos ganando un total de 99gramos. En la necesidad de Respirar normalmente, se observó una mejoría de 2 a 4 puntos en los tres indicadores: estado mental, facilidad de la respiración, ausencia de inquietud, posterior a la intervención de enfermería al identificar el diagnostico, Deterioro del Intercambio gaseoso, observando cambios notorios en cuanto a la facilidad de respirar siendo su frecuencia respiratoria 54 x min al alta de Recién nacido.

Posteriormente se elaboró el plan de cuidados de enfermería para mejorar la atención de los recién nacidos ictericia patológica que son atendidos en el área de neonatología del Hospital General Ambato. Se fundamentaron los conceptos teóricos necesarios para el desarrollo de un estudio sobre recién nacido con Ictericia Patológica, que Observamos en el hospital General Ambato, obteniendo un conocimiento de los cuidados que se debe tener en el recién nacidos ingresados en el área de Neonatología. 
Se definieron los aspectos de un plan de cuidados que se analiza y se visualiza los beneficios de utilizar una correcta técnica de enfermería en el cuidado de los recién nacidos con ictericia Patológica en el Hospital General Ambato, misma que ayudara a una pronta recuperación y evitar de esta manera complicaciones como encefalopatía aguda.

Se logró diseñar un plan de cuidados de enfermería para así determinar los aspectos sobre la eficiencia y la eficacia de las técnicas dirigidas a una mejor atención para el recién nacido que presentan esta patología y así reducir el número de complicaciones que presentan los neonatos.

\section{CONFLICTO DE INTERÉS}

Los autores declaran que no tienen conflicto de interés en la publicación del artículo.

\section{FINANCIAMIENTO}

No monetario.

\section{AGRADECIMIENTO}

A la Universidad Regional Autónoma de los Andes; por apoyar el desarrollo de la investigación.

\section{REFERENCIAS}

1. Perry M, Tan Z, Chen J, Weidig T, Xu W, Cong XS. Neonatal Pain: Perceptions and Current Practice. Crit Care Nurs Clin North Am. 2018;30(4):549-561. doi:10.1016/j.cnc.2018.07.013

2. Maxwell LG, Fraga MV, Malavolta CP. Assessment of Pain in the Newborn: An Update. Clin Perinatol. 2019;46(4):693-707. doi:10.1016/j.clp.2019.08.005 
3. Shaw-Battista J. Systematic Review of Hydrotherapy Research: Does a Warm Bath in Labor Promote Normal Physiologic Childbirth?. J Perinat Neonatal Nurs. 2017;31(4):303-316. doi:10.1097/JPN.0000000000000260

4. Cho ES, Kim SJ, Kwon MS, et al. The Effects of Kangaroo Care in the Neonatal Intensive Care Unit on the Physiological Functions of Preterm Infants, MaternalInfant Attachment, and Maternal Stress. J Pediatr Nurs. 2016;31(4):430-438. doi:10.1016/.j.pedn.2016.02.007

5. Al Maghaireh DF, Abdullah KL, Chan CM, Piaw CY, Al Kawafha MM. Systematic review of qualitative studies exploring parental experiences in the Neonatal Intensive Care Unit. J Clin Nurs. 2016;25(19-20):2745-2756. doi: $\underline{10.1111 / j o c n .13259}$

6. Aagaard H, Uhrenfeldt L, Spliid M, Fegran L. Parents' experiences of transition when their infants are discharged from the Neonatal Intensive Care Unit: a systematic review protocol. JBI Database System Rev Implement Rep. 2015;13(10):123-132. doi:10.11124/jbisrir-2015-2287

2021 por los autores. Este artículo es de acceso abierto y distribuido según los términos y condiciones de la licencia Creative Commons Atribución-NoComercial-Compartirlgual 4.0 Internacional (CC BY-NC-SA 4.0) (https://creativecommons.org/licenses/by-nc-sa/4.0/). 